\title{
Análise da ecoeficência nas operações de terminais portuários com a aplicação de técnica de auxílio multicritério à decisão
}

\author{
Ilton Curty Leal Junior ${ }^{1}$ e Vanessa de Almeida Guimarães ${ }^{2}$
}

\begin{abstract}
Resumo: As atividades em terminais portuários são fundamentais para o desenvolvimento econômico de determinada região ou país. Entretanto, tendem a causar influências ambientais afetando a qualidade do ar, da água e do solo. O objetivo deste trabalho é avaliar o desempenho de portos brasileiros, levando em consideração fatores econômicos e ambientais. Pretende-se obter um ranking dos portos em termos de desempenho em ecoeficiência. Para tanto, foram utilizadas medidas de ecoeficiência que combinam indicadores relacionados ao valor do serviço e influências ambientais. Foram feitas pesquisas bibliográficas, para entendimento dos principais conceitos aplicados ao estudo (atividades portuárias, meio ambiente e avaliação de desempenho por meio da ecoeficiência), e documentais, para levantamento de dados. A avaliação de desempenho foi desenvolvida com a utilização de uma técnica de auxílio multicritério à decisão conhecida como Análise Relacional Grey. Ao final deste artigo foi possível identificar o nível de desempenho dos portos escolhidos e elaborar um ranking com base na ecoeficiência.
\end{abstract}

DOI:10.4237/transportes.v21i3.688.

Palavras-chave: ecoeficiência, terminais portuários, análise relacional grey.

\begin{abstract}
Activities in port terminals are critical to the economic development of a specific region or country. However, these activities cause environmental influences which affect the air, water and soil quality. Considering this fact, the main objective of this paper is to evaluate the Brazilian ports performance taking into consideration economic and environmental factors. Thus, we intended to get a ranking of these ports due to eco-efficiency. So, we used eco-efficiency measures that match value of services and environmental influences indicators. A bibliographic research was made in order to understand the main concepts related to port activities, environment and performance evaluation using eco-efficiency. A documental research was used to obtain relevant data base to this study. The performance evaluation was made using a multicriteria technique decision known as Grey Relational Analysis. By doing this article, we could identify the performance level of the chosen ports and could make a ranking based on eco-efficiency.
\end{abstract}

Keywords: ecoefficiency, port terminals, grey relational analysis.

\section{INTRODUÇÃO}

Os problemas relacionados a questões ambientais trazem à tona a discussão sobre como as atividades portuárias influenciam o meio ambiente. Pela sua importância econômica, um porto deve ser eficiente de forma a produzir cada vez mais movimentações de cargas com o mínimo de recursos empregados para isto. Dessa forma, visando unir os objetivos de eficiência e melhoria do aspecto ambiental, este trabalho aborda um conceito que pode trazer benefícios às atividades portuárias: a ecoeficiência.

Com base neste contexto, este artigo se baseia nas seguintes perguntas para representar a problemática: (1) Como relacionar as atividades de movimentação em portos com o conceito de ecoeficiência? (2) Qual o desempenho em termos de ecoeficiência dos portos brasileiros? Assim, o objetivo principal consiste em analisar o desempenho das operações portuárias, sob o enfoque da ecoeficiência, utilizando a técnica de auxílio multicritério conhecida como Análise Relacional Grey - GRA.

Para isso, têm-se os seguintes objetivos específicos: (1) identificar os indicadores ambientais relacionados ao ambiente portuário; (2) estabelecer as medidas de ecoeficiência

\footnotetext{
${ }^{1}$ Ilton Curty Leal Junior, Universidade Federal Fluminense, Volta Redonda, RJ, Brasil. (e-mail: iltoncurty@gmail.com)

${ }^{2}$ Vanessa de Almeida Guimarães, Universidade Federal do Rio de Janeiro, Rio de Janeiro, RJ, Brasil. (e-mail: vanessaguim@hotmail.com)
}

Manuscrito recebido em 16/09/2013 e aprovado para publicação em 06/10/2013. Este artigo é parte de TRANSPORTES v. 21, n. 3, 2013. ISSN: 2237-1346 (online). DOI:10.4237/transportes.v21i3.688. para a aplicação da GRA; (3) estabelecer um ranking do desempenho global em termos de ecoeficiência de cinco portos brasileiros. São eles: Rio Grande, Santos, Suape, Paranaguá/Antonina e Belém. Os quatro primeiros portos são os de maior movimentação de carga e o quinto porto representa a região norte do Brasil. Além disso, selecionaram-se tais portos por não terem sido encontradas bases de dados que permitissem a análise dos demais.

A partir desta introdução este artigo apresenta a seção 2, que aborda o referencial teórico, a seção 3 que trata da metodologia utilizada e a seção 4 que faz uma aplicação e análise da ecoeficiência em terminais portuários. A seção 5 apresenta as conclusões, limitações e proposições para novos estudos e por fim, apresenta-se a lista de referências bibliográficas.

\section{REFERENCIAL TEÓRICO}

Nesta seção são apresentados os principais conceitos que deram suporte a realização deste trabalho.

\subsection{Ecoeficiência em operação portuária}

Em geral, considera-se que levar em conta atributos ambientais no processo decisório é uma prática diretamente associada ao aumento de custos, e, portanto, é prejudicial aos objetivos de lucro e rentabilidade de uma organização. O conceito de ecoeficiência, no entanto, demonstra que é possível combinar conceitos, aparentemente antagônicos, de desempenho econômico e de redução de influências ambientais (Leal Jr., 2010). 
Segundo o World Business Council for Sustainable Development (WBCSD, 2000 e 2006), a ecoeficiência consiste na entrega de bens e serviços, a preços competitivos, que ao mesmo tempo: satisfaçam as necessidades humanas, resultem em melhor qualidade de vida e reduzam, progressivamente, as influências ambientais e a intensidade do uso dos recursos em todas as etapas do ciclo de produção do bem ou serviço. Portanto, esta abordagem enfoca a utilização adequada de recursos materiais e energéticos com o sentido de se reduzir custos e/ou de se maximizar lucros.

A utilização do conceito de ecoeficiência para avaliação do desempenho requer medidas específicas as quais estão baseadas na relação entre o valor do produto (bem ou serviço) e a influência ambiental, conforme representado na Equação 1.

$$
\text { Ecoef }=\frac{V B S}{I A}
$$

em que Ecoef é o índice de ecoeficiência, VBS é o valor do bem ou serviço e IA representa as influências ambientais.

Destaca-se que esta equação foi proposta pelo WBCSD para estudos que adotem medidas de ecoeficiência. A relevância desta equação está no fato de que a mesma considera uma relação entre ganhos econômicos e redução de influências ambientais para melhorar o valor da ecoeficiência, conforme propõe o artigo.

Analisando a Equação 1 verifica-se que um aumento no valor do produto (bem ou serviço) e/ou a redução de influências ambientais reflete positivamente na medida de ecoeficiência e, consequentemente, no desempenho empresarial. Obtém-se um maior desempenho por se tratar de uma relação direta entre o quanto se produz (representados pelo valor do produto ou serviço) e os recursos utilizados (representados pelas influências ambientais).

Sendo assim, a utilização de medidas de ecoeficiência requer algumas investigações nos processos produtivos, a fim de detectar ineficiências e falhas, bem como diminuir os desperdícios (Michelini et al., 2004).

O WBCSD (2000) apresenta uma metodologia para avaliação da ecoeficiência que pode ser aplicada pelas organizações. De forma complementar, o trabalho de Leal $\mathrm{Jr}$ (2010) propõe uma estrutura, composta de aspectos, atributos e indicadores para avaliação de desempenho em transportes, com base na ecoeficiência. Cada aspecto é composto por um conjunto de atributos que, por sua vez, são compostos por indicadores, os quais podem ser usados para construir medidas de ecoeficiência, conforme exposto na Tabela 1 .

Analisando as fontes consultadas para realização deste trabalho, percebe-se que a ecoeficiência não é usualmente abordada no setor de transporte, estando presente, principalmente, na área industrial. A British Petroleum (BP, 2003), por exemplo, aborda a ecoeficiência na redução do consumo de recursos naturais (como energia, materiais e água), promovendo a reciclagem e redução dos desperdícios. Enquanto o WBCSD (2000) trabalha com a questão da revalorização dos subprodutos, a partir do uso da "bolsa de resíduos” onde são negociados os rejeitos de uma empresa para aproveitamento por outras empresas.

Ainda, Martins et al. (1999) analisam a inclusão de programas de gestão de transporte rodoviário, considerando as metas de uso eficiente de combustível, o que envolve o con- trole de tráfego, a formação dos condutores e a manutenção dos veículos. Santos (2001) analisa os sistemas de gestão de qualidade, saúde ocupacional, segurança e meio ambiente levando em conta a aplicação dos conceitos de ecoeficiência e seus benefícios econômicos e ambientais.

Já em PETROBRAS (2003) é possível observar a aplicação dos conceitos de ecoeficiência para a realização de investimentos na utilização de energia alternativa; na gestão do consumo de energia e de água; na redução de emissão de dióxido de carbono, óxidos de enxofre e de material particulado; na redução do volume de vazamentos de óleo, resíduos perigosos, não perigosos e efluentes líquidos e na diminuição da incidência de acidentes com vazamentos.

Pinho, Catanzano e Candeira (2011), por sua vez, abordam questões ligadas ao gerenciamento ambiental por meio de um sistema de ecoeficiência, cujas ferramentas têm como principal função a preservação do meio ambiente e o controle de perdas dos processos. Leyen (2008) avalia a aplicação dos conceitos de ecoeficiência na exploração e produção de petróleo e gás em regiões de florestas tropicais úmidas. Já Michelini et al. (2004) sugerem a aplicação de indicadores de ecoeficiência para avaliação do setor termoelétrico brasileiro a carvão mineral enquanto Hendriks e Jager (2001) aplicam este conceito ao transporte de gás natural por gasoduto.

O Global Reporting Initiative (GRI, 2000) apresenta a elaboração de relatórios de sustentabilidade incluindo três elementos: (1) econômico, relacionado à produtividade e à redução de gastos com a produção; (2) ambiental, incluindo os impactos dos processos produtivos no meio ambiente e na saúde humana e (3) social, abordando a saúde e a segurança do empregado.

Nessa vertente, Cantarino (2003) apresenta a aplicação da ecoeficiência na avaliação de desempenho ambiental e na formulação de políticas públicas. Por fim, Cheon et al. (2009) avaliam os impactos das reformas institucionais sobre as mudanças da eficiência dos portos abordando os conceitos de ecoeficiência.

Em relação especificamente à operação portuária, foram realizados levantamentos bibliográficos e documentais, selecionando-se os terminais de contêineres internacionais com maior taxa de movimentação (Noteboom, 2004; Journal of Commerce - JOC, 2012), dos quais se analisaram os relatórios publicados em seus sites.

Dos sessenta portos pesquisados, apenas 30\% publicam relatórios ambientais, sendo que somente quatro (três americanos e um canadense) elaboram inventários completos de emissões de gases de efeito estufa (GGE) e poluentes atmosféricos. Os demais apenas publicam informações sobre consumo e descarte de material. Nenhum desses relatórios, porém, utiliza o conceito de ecoeficiência relacionando o valor do bem ou serviço com influências ambientais. Esse número reduzido de referências sugere carência de estudos na área de transportes, principalmente no setor portuário.

No Brasil, a Agência Nacional de Transportes Aquaviário (ANTAq) vem indicando medições e monitoramentos da qualidade ambiental com o intuito de estabelecer políticas para redução dos impactos ambientais dos portos. Por meio de alguns relatórios a ANTAq orienta os agentes por ela regulados a exercerem suas atividades com responsabilidade ambiental (ANTAq, 2011a). Contudo, nos relatórios da ANTAq investigados neste trabalho, não foram encontrados valores quantitativos de impactos ambientais, nem a utiliza- 
Tabela 1. Aspectos, Atributos e Indicadores relacionados ao conceito de ecoeficiência (com base em Leal Jr (2010))

\begin{tabular}{|c|c|c|c|}
\hline Aspectos & Atributos & Indicadores & Unidade \\
\hline \multirow{5}{*}{ 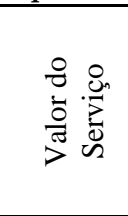 } & \multirow{2}{*}{ Valor Monetário } & Valor monetário do volume de carga & US\$ \\
\hline & & Receita líquida do terminal portuário & US\$ \\
\hline & \multirow{3}{*}{ Serviço Produzido } & Número de contêineres movimentados & TEUs \\
\hline & & Volume movimentado & $\mathrm{m}^{3}$ \\
\hline & & Quantidade movimentada & $\mathrm{t}$ \\
\hline \multirow{36}{*}{ 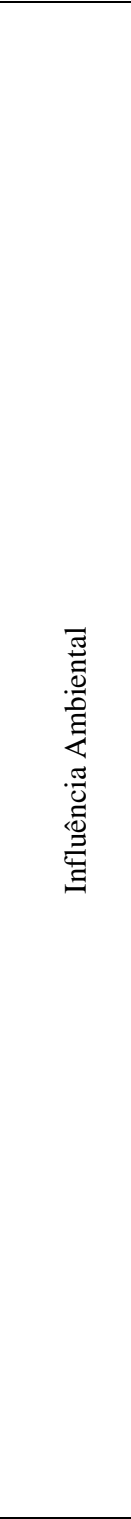 } & \multirow{3}{*}{$\begin{array}{l}\text { Consumo de } \\
\text { Energia }\end{array}$} & Consumo total de energia & MJ \\
\hline & & Consumo de energia elétrica & MJ \\
\hline & & Consumo total de energia renovável & MJ \\
\hline & \multirow{2}{*}{ Segurança } & Número de acidentes nas movimentações de carga & qtd \\
\hline & & Custo total com acidentes & US\$ \\
\hline & \multirow{4}{*}{$\begin{array}{l}\text { Emissão de Gases } \\
\text { de Efeito Estufa }\end{array}$} & Emissão de gás $\mathrm{CO}_{2}$ & $\mathrm{~kg}$ \\
\hline & & Emissão de vapor de $\mathrm{H}_{2} \mathrm{O}$ & $\mathrm{kg}$ \\
\hline & & Emissão de gás metano & $\mathrm{kg}$ \\
\hline & & Emissão de CFC & $\mathrm{kg}$ \\
\hline & \multirow{6}{*}{$\begin{array}{l}\text { Poluição } \\
\text { Atmosférica }\end{array}$} & Emissão de hidrocarbonetos & $\mathrm{kg}$ \\
\hline & & Emissão de aldeídos & $\mathrm{kg}$ \\
\hline & & Emissão de gás CO & $\mathrm{kg} / \mathrm{ppb}$ \\
\hline & & Emissão de $\mathrm{N}_{2} \mathrm{O}$ & $\mathrm{kg}$ \\
\hline & & Emissão de gases acidificantes (SOx, NOx) & $\mathrm{kg}$ \\
\hline & & Emissão de materiais particulados & $\mathrm{kg}$ \\
\hline & \multirow{10}{*}{$\begin{array}{l}\text { Poluição da Água } \\
\text { e do Solo }\end{array}$} & Quantidade descartada de óleo pelos equipamentos de movimentação & $\mathrm{l}$ \\
\hline & & Descarte de lubrificantes & l \\
\hline & & Vazamento de produtos químicos & l \\
\hline & & Geração de resíduos sólidos & $\mathrm{t}$ \\
\hline & & Descarte de água de lastro & $\mathrm{l}$ \\
\hline & & Salinidade da água & $\mathrm{ppm}$ \\
\hline & & Hidrocarbonetos em sedimentos & Adimensional \\
\hline & & Não atendimentos a normas referentes a resíduos sólidos & $\%$ \\
\hline & & Sedimentos com metais pesados & Adimensional \\
\hline & & Geração de esgoto (águas cinzas) & 1 \\
\hline & \multirow{3}{*}{ Poluição Sonora } & Intensidade total de ruído emitido pelos equipamentos de movimentação & $\mathrm{Db}$ \\
\hline & & Intensidade total de ruído emitido pelos veículos que chegam ao porto & $\mathrm{Db}$ \\
\hline & & Intensidade de ruído de equipamentos de movimentação & $\mathrm{Db}$ \\
\hline & \multirow{2}{*}{ Poluição Térmica } & Quantidade de calor liberado & MJ \\
\hline & & Volume de efluentes superaquecidos descarregados no ambiente aquático & $\mathrm{m}^{3}$ \\
\hline & \multirow{2}{*}{ Poluição Visual } & Espaço ocupado pelos portos nas cidades & $\mathrm{km}^{2}$ \\
\hline & & Área total disponível & $\mathrm{km}^{2}$ \\
\hline & \multirow{2}{*}{ Consumo de Água } & Consumo de água em operação & 1 \\
\hline & & Volume de água reutilizado & $\mathrm{l}$ \\
\hline & \multirow{2}{*}{$\begin{array}{l}\text { Consumo de } \\
\text { Material }\end{array}$} & Quantidade descartada provenientes de peças de reposição & $\mathrm{kg}$ \\
\hline & & Consumo/descarte de aço, plástico, madeira, papel na operação & $\mathrm{kg}$ \\
\hline
\end{tabular}

ção de medidas de ecoeficiência.

\subsection{Técnicas multicritério de apoio à decisão}

As técnicas de apoio multicritério a decisão começaram a surgir a partir da década de 1970 com o intuito de ajudar na resolução problemas associados à tomada de decisão. Como vantagens da abordagem multicritério podem-se citar: (1) constituição de uma base de diálogo utilizando diversos pontos de vista comuns; (2) maior facilidade de incorporar incertezas; (3) enfrentar cada solução como um compromisso de objetivos em conflito (Gomes, 2004).

Segundo Malczewiski (1999) a tomada de decisão multicritério se divide em duas classes: 1) tomada de decisão multiobjetivo e 2) tomada de decisão multiatributo. A primeira é aplicada a problemas que têm objetivos múltiplos, nos quais as alternativas podem adquirir um número infinito de valores. Neste caso, o objetivo é considerado em uma função de maximização ou minimização com base em variáveis ou atributos considerados. A melhor solução se encontra dentro de uma região de soluções viáveis (Malczewiski, 1999). Os métodos com esta característica fazem parte do ramo contínuo da decisão multicritério (Gomes, 2004).

Já na segunda, tem-se um conjunto de alternativas de decisão formada por um número finito e geralmente pequeno de variáveis. Nos problemas de decisão multiatributo, a solução consiste em encontrar a melhor alternativa baseada em seus atributos. Aqui se encontram os métodos do ramo discreto da decisão multicritério (Gomes, 2004).

\subsubsection{Análise Relacional Grey}

Dentre as diversas técnicas disponíveis este trabalho utiliza a técnica multiatributo conhecida como Análise Relacional Grey (GRA) que faz parte da teoria dos sistemas grey. Esta 
Tabela 2. Aplicação da Análise Relacional Grey na área de transportes

\begin{tabular}{ll}
\hline Referência & Aplicação \\
\hline Wen (2004) & Avaliação da qualidade dos serviços das linhas aéreas em Taiwan \\
\hline Garcia et al. (2005) & Avaliação e escolha de fornecedores e transportadores \\
\hline Leal Jr e Garcia (2008) & Escolha de modos de transporte de carga para produtos perigosos \\
\hline $\begin{array}{l}\text { Leal Jr e D’Agosto (2009) } \\
\text { Leal Jr e D’Agosto (2011) }\end{array}$ & Escolha de modos de transporte de carga \\
\hline Wang et al. (2004) & Análise comparativa da performance operacional dos aeroportos de Taiwan \\
\hline Yang e Chen (2005) & Seleção de fornecedores \\
\hline Lu e Wevers (2007) & Avaliação de medidas de segurança no tráfego rodoviário \\
\hline Pai et al. (2007) & Avaliação dos efeitos do transporte na qualidade do ar \\
\hline Qu et al. (2007) & Previsão de volume de frete \\
\hline Lo et al. (2009) & Avaliação de modelos de entrega rodoviários \\
\hline Hsu et al. (2009) & Gestão de segurança aérea \\
\hline Cheon et al. (2009) & $\begin{array}{l}\text { Avaliação dos impactos das reformas institucionais sobre as mudanças da } \\
\text { eficiência dos portos }\end{array}$ \\
\hline Leal Jr (2010) & Escolha modal no transporte de produtos perigosos \\
\hline
\end{tabular}

teoria foi proposta por Julong Deng, em 1982, com o intuito de evitar os problemas inerentes aos métodos estatísticos e poder utilizar uma quantidade limitada de dados para estimar o comportamento de um sistema incerto (Wen, 2004).

A teoria grey tem sido aplicada nos mais variados campos de pesquisa como produção, sistemas sociais, ecologia, economia, geografia, tráfego, gerenciamento e educação. Segundo Bischoff (2008) a GRA utiliza a informação do sistema grey para comparar dinamicamente cada fator, de forma quantitativa.

A Análise Relacional Grey pode ser usada na área de transportes e na Tabela 2 apresentam-se os trabalhos pesquisados e suas respectivas aplicações.

A modelagem matemática da Análise Relacional Grey utilizada nas pesquisas levantadas está apresentada a seguir. Seja um conjunto de observações $\left\{x_{0}{ }^{(0)}, x_{1}{ }^{(0)}, \ldots x_{m}{ }^{(0)}\right\}$, onde $x_{0}{ }^{(0)}$ é uma observação referencial e $x_{1}{ }^{(0)}, x_{2}{ }^{(0)}, \ldots, x_{m}{ }^{(0)}$ são observações originais a serem comparadas. Cada observação $x_{i}$ possui $n$ medidas que são descritas sob a forma de séries $x_{i}=\left\{x_{i}^{(0)}(k), \ldots x_{m}{ }^{(0)}(n)\right\}$, onde cada componente dessa série, antes de qualquer operação, é normalizado da forma a seguir sendo, $x_{i}^{(0)}(k)$ o valor de uma medida $k$ para uma observação original $x_{i}^{(0)}$. 2).

Se quanto maior o valor da observação, melhor (Equação

$$
\begin{gathered}
x_{i}^{\prime}(k)=\frac{x_{i}^{(o)}(k)-\min _{\forall i}\left(x_{i}^{(o)}(k)\right)}{\max _{\forall i}\left(x_{i}^{(o)}(k)\right)-\min _{\forall i}\left(x_{i}^{(o)}(k)\right)} \\
\text { para i }=0,1,2, . ., \mathrm{m}, \quad \mathrm{k}=1,2, \ldots, \mathrm{n}
\end{gathered}
$$

Se quanto menor o valor da observação, melhor (Equação 3).

$$
\begin{gathered}
x_{i}^{\prime}(k)=\frac{\max _{\forall i}\left(x_{i}^{(o)}(k)\right)-x_{i}^{(o)}(k)}{\max _{\forall i}\left(x_{i}^{(o)}(k)\right)-\min _{\forall i}\left(x_{i}^{(o)}(k)\right)} \\
\text { para i }=0,1,2, . ., \mathrm{m}, \quad \mathrm{k}=1,2, \ldots, \mathrm{n}
\end{gathered}
$$

em que $x_{i}^{\prime}(k)$ é o valor normalizado de uma medida $k$ para uma observação original $x_{i}^{(0)}$.

A série cujos atributos normalizados são os melhores possíveis e representa o estado desejado para qualquer série é representada por $x_{0}$, sendo os valores da mesma igualados a 1. Essa é uma abordagem que propõe um contorno para os muitos casos em que a medida de referência não é facilmente encontrada ou difícil de ser calculada.

Após a normalização dos dados de cada série, calculamse os coeficientes relacionais grey $\gamma$ (Equação 4).

$$
=\frac{\min _{\forall i} \min _{\forall k}\left|x_{0}(k)-x_{1}(k)\right|+\zeta \max _{\forall i} \max _{\forall k}\left|x_{0}(k)-x_{1}(k)\right|}{x_{0}(k)-x_{1}(k)+\zeta \max _{\forall i} \max _{\forall k}\left|x_{0}(k)-x_{1}(k)\right|}
$$

em que $\zeta \in[0,1]$ assume, em geral, o valor 0,5 , sendo útil somente para diferenciar os elementos da série e não influenciando na ordenação final das séries (Deng, 1989).

Depois de estabelecidos os coeficientes relacionais grey, devem-se estabelecer os graus de relacionamento grey $\left(\Gamma_{i}\right)$ para cada série de dados (Deng, 1989), conforme a Equação 5, que é a média aritmética simples dos coeficientes relacionais grey para cada alternativa.

$$
\Gamma_{i}=\frac{1}{n} \sum_{k=1}^{n} \gamma_{i}\left(x_{0}^{\prime}(k), x_{i}^{\prime}(k)\right)
$$

Os graus de relacionamento grey podem ser calculados ponderando os coeficientes relacionais grey. Neste caso os pesos podem ser, por exemplo, atribuídos por especialistas consultados ou simplesmente arbitrados pelo avaliador. A Equação 6 é utilizada para calcular a média ponderada.

$$
\Gamma_{i}=\sum_{k=1}^{n} \beta_{k} \gamma_{i}\left(x_{0}^{\prime}(k), x_{i}^{\prime}(k)\right)
$$

em que $\beta_{\mathrm{k}}$ é o peso de cada atributo e $\sum_{j=1}^{n} \beta_{k}=1$.

No caso da utilização das medidas ecoeficiência, os pesos devem ser dados às medidas. Se o avaliador dispuser dos pesos dos atributos de influência ambiental, os mesmos poderão ser usados para as respectivas medidas, já que o peso do atributo de valor do bem ou serviço será anulado pelo fato de serem os mesmos em cada medida formada.

Por meio da Equação 6 é possível realizar análise de sensibilidade alterando-se os pesos das medidas, desde que o 
somatório das mesmas continue sendo igual a 1. Na análise de sensibilidade selecionam-se valores de variáveis utilizadas para serem alteradas e assim observar qual o comportamento dos resultados do modelo (Taha, 2008). Após o cálculo dos graus de relacionamentos grey, ranqueiam-se as sequências, sendo esse procedimento chamado Ranking Relacional Grey.

\section{METODOLOGIA}

Para a realização deste artigo foram utilizados dois tipos de pesquisas: bibliográfica e documental. Por meio de pesquisa bibliográfica foi possível entender os conceitos relacionados ao estudo, levantar os atributos, indicadores e medidas importantes para a avaliação da ecoeficiência e elencar fontes de dados relevantes acerca do assunto. Além disso, permitiu conhecer uma seleção de métodos de avaliação de desempenho relativos à ecoeficiência.

Com este entendimento teórico foi possível realizar a coleta de dados, por meio de pesquisa documental, junto a ANTAq e aos demais órgãos de administração portuária regional.

Após a tabulação dos dados, os mesmos foram utilizados para se estabelecer um ranking dos portos segundo o desempenho em termos de ecoeficiência. Para isto foi utilizada a técnica de auxílio multicritério Análise Relacional Grey que se baseou nas medidas de ecoeficiência (Equação 1) formadas a partir dos indicadores apresentados na Tabela 1.

Em seguida, para melhor entendimento das relações entre as medidas e o desempenho dos portos, foi realizada uma análise de sensibilidade que considerou a variação dos indicadores utilizados para compor as medidas de ecoeficiência. Assim, foi possível estabelecer cenários para comparar o desempenho dos portos estudados. Além disso, foi realizada uma segunda análise de sensibilidade considerando a variação nos pesos das medidas, os quais foram obtidos aplicando-se a Equação 6 da Análise Relacional Grey.
Por fim, após a análise dos resultados, foram apresentadas as conclusões do artigo, suas limitações e algumas recomendações de novos estudos que complementem ou melhorem os resultados encontrados com a pesquisa realizada.

\section{ANÁLISE DE ECOEFICIÊNCIA DAS OPERAÇÕES PORTUÁRIAS}

A aplicação proposta neste trabalho se limitou a cinco portos por não terem sido encontrados documentos e estatísticas que fornecessem dados a respeito de indicadores ambientais para todos os portos brasileiros. Valois (2009) apresenta os valores de indicadores ambientais (que constam na tabela 1) para os cinco portos escolhidos, conforme Tabela 3.

A quantidade movimentada (em toneladas) foi escolhida como variável de valor de serviço, conforme recomendado por WBCSD (2000), que indica não serem necessários valores monetários para representar este aspecto. Outro motivo para esta escolha foi o fato destes valores estarem disponíveis para consulta nos sites dos portos e nos relatórios da ANTAq.

A partir destes indicadores, foram calculadas as medidas para cada porto (com base na Equação 1), cujos resultados estão apresentados na Tabela 4.

As medidas foram normalizadas, conforme a Equação 2, que prevê que os valores das medidas são melhores quanto maiores forem, já que se espera que a ecoeficiência de cada uma delas seja a maior possível, considerando as séries normalizadas, $x ` i(k)$, com $i=0, \ldots, 5$ e $k=1, \ldots, 5$, como sendo representantes das alternativas $i$ e de suas medidas $k$.

O desempenho global foi calculado aplicando-se primeiro a Equação 4 para estabelecer o coeficiente relacional grey, admitindo $\zeta=0,5$. Como essa aplicação inicial não prevê a utilização de pesos associados às medidas, utilizouse a Equação 5 para apurar os graus de relacionamento grey, apresentados na Figura 1.

Ao analisar os resultados constata-se a superioridade do

Tabela 3. Indicadores utilizados para avaliação da ecoeficiência dos portos [Fontes: VALOIS (2009), Companhia Docas do Pará - CDP (2011), ANTAq (2011b)]

\begin{tabular}{|c|c|c|c|c|c|c|}
\hline Porto & $\begin{array}{l}\text { Quantidade } \\
\text { Movimentada } \\
\text { IVS* } \\
\text { [t] }\end{array}$ & $\begin{array}{l}\text { Salinidade } \\
\text { da água } \\
\text { IA1 } \\
\text { [ppm] }\end{array}$ & $\begin{array}{l}\text { Sedimentos com } \\
\text { metais pesados } \\
\text { IA2 } \\
\text { [Adimensional] }\end{array}$ & $\begin{array}{l}\text { Hidrocarbonetos } \\
\text { em sedimentos } \\
\text { IA3 } \\
\text { [Adimensional] }\end{array}$ & $\begin{array}{l}\text { Emissão } \\
\text { CO } \\
\text { IA4 } \\
{[p p b]} \\
\end{array}$ & $\begin{array}{l}\text { Não atendimentos a } \\
\text { normas referentes a } \\
\text { resíduos sólidos } \\
\text { IA5 } \\
\text { [\%] }\end{array}$ \\
\hline Rio Grande & 23.908 .887 & 35,00 & 6,00 & 1,16 & 325,000 & 0,250 \\
\hline Santos & 83.194.129 & 33,00 & 2,00 & 4,50 & 225,000 & 0,500 \\
\hline Suape & 7.736 .622 & 36,00 & 2,00 & 1,08 & 137,500 & 0,010 \\
\hline Belém & 20.580 .704 & 35,00 & 3,00 & 5,85 & 162,500 & 0,750 \\
\hline Paranaguá/ Antonina & 31.200 .000 & 36,00 & 4,00 & 1,05 & 625,000 & 1,000 \\
\hline
\end{tabular}

Tabela 4. Medidas utilizadas para avaliação da ecoeficiência dos portos

\begin{tabular}{|c|c|c|c|c|c|}
\hline Alternativas & $\begin{array}{l}\text { M1 } \\
\text { IVS/IA1 } \\
{[t] /[p p m]} \\
\end{array}$ & $\begin{array}{l}\text { M2 } \\
\text { IVS/IA2 } \\
\text { [t]/[Adimensional] }\end{array}$ & $\begin{array}{l}\text { M3 } \\
\text { IVS/IA3 } \\
\text { [t]/[Adimensional] }\end{array}$ & $\begin{array}{l}\text { M4 } \\
\text { IVS/IA4 } \\
{[t] /[p p b]} \\
\end{array}$ & $\begin{array}{l}\text { M5 } \\
\text { IVS/IA5 } \\
{[t] /[\%]} \\
\end{array}$ \\
\hline Rio Grande & $683.111,06$ & $3.984 .814,50$ & $2.0611 .109,48$ & $73.565,81$ & $95.635 .548,00$ \\
\hline Santos & $2.521 .034,21$ & $41.597 .064,50$ & $18.487 .584,22$ & $369.751,68$ & $166.388 .258,00$ \\
\hline Suape & 214.906,17 & 3.868.311,00 & 7.196.857,67 & $56.266,34$ & $773.662 .200,00$ \\
\hline Belém & $588.020,11$ & $6.860 .234,67$ & 3.518.069,06 & $126.650,49$ & $27.440 .938,67$ \\
\hline Paranaguá/Antonina & $866.666,67$ & $7.800 .000,00$ & 29.714.285,71 & $49.920,00$ & $31.200 .000,00$ \\
\hline
\end{tabular}

Nota: M1 a M5 representam as medidas formadas pelo indicador de valor de serviço divido por cada uma das influências ambientais escolhidas. 


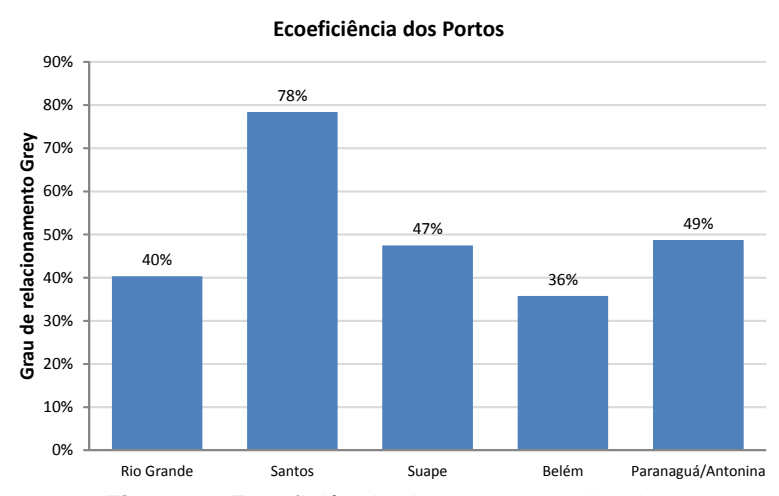

Figura 1. Ecoeficiência dos portos analisados

desempenho do porto de Santos em relação aos demais. Isto pode ser explicado pelo fato da sua movimentação, em 2009, ter sido praticamente igual à soma da movimentação dos demais portos analisados, ao passo que seu nível de influências ambientais ficou abaixo da média (com exceção dos hidrocarbonetos em sedimentos que apresentou o valor mais alto).

Como os indicadores analisados são referentes ao ano de 2009, uma nova análise foi feita a fim de se identificar se haveria modificação no ranking. Nesta, adotaram-se os valores de movimentação referentes ao ano de 2011 (Tabela 5), contudo, considerou-se que os impactos ambientais se mantiveram nos mesmos níveis apresentados em 2009. Aplicando-se o procedimento da análise relacional grey, observou-se que não houve mudanças significativas de desempenho. Mesmo o porto de Suape, que teve um aumento de $42 \%$ em sua movimentação, manteve-se na mesma posição no ranking.

Esses resultados mostram que para que os demais portos consigam melhorar seus desempenhos (comparados uns aos outros), eles deveriam aumentar sua movimentação aos níveis do Porto de Santos e/ou, ainda, reduzir seus impactos ambientais. Por meio da análise dos dados observa-se que uma maior movimentação de carga em um porto, não necessariamente aumenta suas as influências ambientais.

Identificou-se, ainda, que o porto de Belém apresentou o desempenho mais baixo dentre os portos estudados, em ambas as análises (2009 e 2011). Assim, a fim de aumentar sua ecoeficiência, foi feita uma análise considerando três cená- rios: (1) redução das influências ambientais aos níveis mais baixos encontrados na análise original (com base na Tabela 3); (2) aumento da movimentação em $42 \%$, conforme observado no porto de Suape entre 2009 e 2011 e (3) a combinação dos dois cenários anteriores. Os resultados de desempenho no ranking podem ser observados na Tabela 6 . Considerou-se que os demais portos permaneceram com os mesmos valores de movimentação e influências ambientais.

Verifica-se que, para o porto de Belém, a redução das influências ambientais (cenário 1) proporcionou melhor desempenho do que o aumento na quantidade movimentada (cenário 2), mas que foi o cenário 3 (que combina os dois anteriores) que a alcançou o melhor resultado. Entretanto, apesar de atingir um melhor desempenho no cenário 3, a posição no ranking se manteve inalterada em relação ao primeiro cenário, permanecendo em segundo lugar.

Assim, para se decidir quais ações devem ser tomadas tendo em vista a melhoria da ecoeficiência, é necessário um estudo mais aprofundado sobre a viabilidade das mesmas. Para o porto de Belém melhorar seu desempenho no cenário 1 , por exemplo, seria necessário reduzir a salinidade da água em $6 \%$, os sedimentos com metais pesados em $33 \%$, os hidrocarbonetos em sedimentos em $82 \%$, a emissão de CO em 15\% e o percentual de não atendimento a normas referentes a resíduos sólidos em 99\%.

O aumento de movimentação no cenário 2 pode estar associado a atração de maior demanda para o porto, considerando que o mesmo ainda não atingiu sua capacidade produtiva, e isso irá depender de fatores econômicos e políticos. Por outro lado, aumentar a movimentação pode estar relacionado a investimentos em capacidade, que dependerá também da administração do porto.

Como uma medida de ecoeficiência pode ser considerada mais importante do que as demais, em função dos pesos atribuídos a cada uma delas, realizou-se uma análise de sensibilidade, utilizando-se a Equação 6. Para isso, foram feitas seis combinações de pesos (CP) diferentes, apresentadas na Tabela 7.

Os resultados são apresentados na Figura 2, que mostra diferentes resultados de desempenho (Graus de Relacionamento Grey) para as diferentes combinações de pesos. Deve-se ressaltar que estes pesos foram arbitrados para realização da análise.

Tabela 5. Desempenho dos portos em função de suas movimentações em 2011 [Fonte: CDP (2011), ANTAq (2011b)]

\begin{tabular}{llll}
\hline & $\begin{array}{l}\text { Quantidade } \\
\text { movimentada } \\
\text { em 2011 [t] }\end{array}$ & $\begin{array}{l}\text { Variação em relação } \\
\text { à movimentação de } \\
\text { cargas em 2009 }\end{array}$ & $\begin{array}{l}\text { Grau de } \\
\text { relacionamento Grey }\end{array}$ \\
\hline Porto & 17.900 .000 & $-25 \%$ & $36 \%$ \\
Sio Grande & 86.000 .000 & $3 \%$ & $77 \%$ \\
Suape & 11.000 .000 & $42 \%$ & $48 \%$ \\
Belém & 19.434 .814 & $-6 \%$ & $35 \%$ \\
Paranaguá/Antonina & 41.000 .000 & $31 \%$ & $50 \%$ \\
\hline
\end{tabular}

Tabela 6. Desempenho dos portos nos três cenários analisados

\begin{tabular}{llll}
\hline \multirow{2}{*}{ Portos } & \multicolumn{3}{l}{ Graus de Relacionamento Grey } \\
\cline { 2 - 4 } & Cenário 1 & Cenário 2 & Cenário 3 \\
\hline Rio Grande & $39 \%$ & $40 \%$ & $39 \%$ \\
Santos & $77 \%$ & $78 \%$ & $77 \%$ \\
Suape & $36 \%$ & $47 \%$ & $35 \%$ \\
Belém & $54 \%$ & $38 \%$ & $64 \%$ \\
Paranaguá/Antonina & $49 \%$ & $49 \%$ & $49 \%$ \\
\hline
\end{tabular}


Tabela 7. Combinações de pesos para a análise de sensibilidade

\begin{tabular}{llrrrrr}
\hline \multirow{2}{*}{$\begin{array}{l}\text { Combinações de } \\
\text { Pesos }\end{array}$} & \multicolumn{2}{l}{ Pesos das medidas } & & & \\
\cline { 2 - 5 } M1 & M2 & M3 & M4 & M5 & Total \\
\hline CP 0 & $20 \%$ & $20 \%$ & $20 \%$ & $20 \%$ & $20 \%$ & $100 \%$ \\
CP 1 & $20 \%$ & $40 \%$ & $20 \%$ & $10 \%$ & $100 \%$ \\
CP 2 & $10 \%$ & $10 \%$ & $20 \%$ & $20 \%$ & $40 \%$ & $100 \%$ \\
CP 3 & $40 \%$ & $20 \%$ & $20 \%$ & $10 \%$ & $10 \%$ & $100 \%$ \\
CP 4 & $30 \%$ & $15 \%$ & $10 \%$ & $15 \%$ & $30 \%$ & $100 \%$ \\
CP 5 & $50 \%$ & $0 \%$ & $0 \%$ & $0 \%$ & $50 \%$ & $100 \%$ \\
CP 6 & $0 \%$ & $40 \%$ & $20 \%$ & $40 \%$ & $0 \%$ & $100 \%$ \\
\hline
\end{tabular}

Nota: (1) combinação de pesos iguais conforme Figura 1.

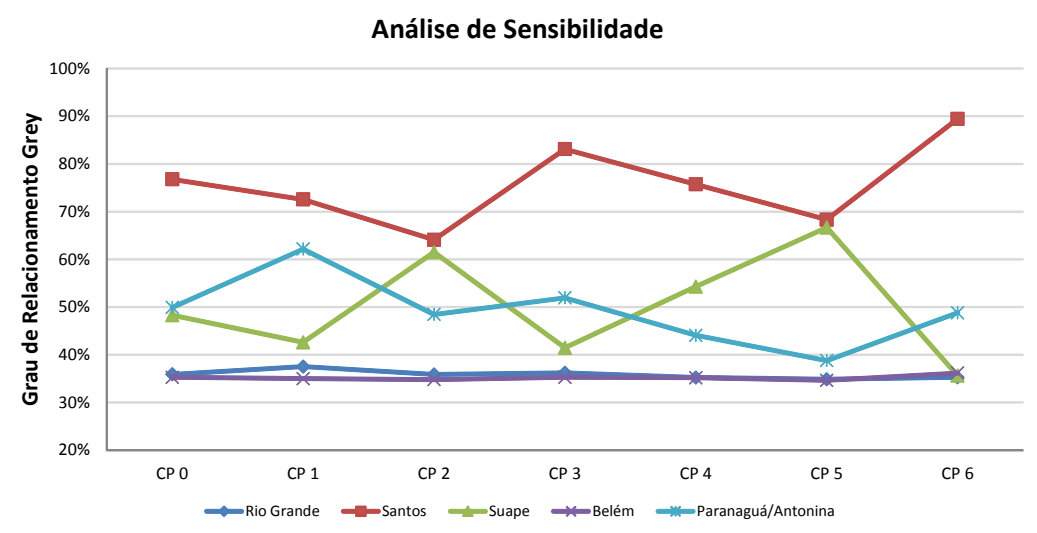

Figura 2. Análise de sensibilidade do desempenho dos portos investigados

Analisando a Figura 2, percebe-se que o porto de Santos apresenta desempenho superior aos demais em todas as CPs. Destaca-se que na CP 1 o desempenho do porto de Paranaguá alcança seu maior índice, em função de ter sido atribuído peso maior a medida em que este se destaca (hidrocarbonetos em sedimentos). Semelhante comportamento acontece com o Porto de Suape nas CPs 2 e 5, cujos desempenhos se aproximam do porto de Santos. Isso ocorreu devido a maior importância atribuída as medidas que envolvem os hidrocarbonetos em sedimentos, emissão de CO e gerenciamento de resíduos sólidos. Os portos de Rio Grande e Belém não apresentaram melhora de desempenho com as combinações de peso realizadas e permaneceram nos últimos lugares em toda a análise.

\section{CONCLUSÃO}

Em função dos dados levantados e da análise realizada é possível responder as perguntas da problemática. Com base no referencial teórico e na aplicação desenvolvida neste artigo foi possível relacionar as atividades empresariais de movimentação em portos com o conceito de ecoeficiência. O desempenho em termos de ecoeficiência dos portos brasileiros foi parcialmente realizado em função de não ter sido possível levantar dados de impactos ambientais para todos eles. Mesmo com a aplicação restrita a cinco portos concluí-se que a sistemática utilizada permitiria a avaliação em âmbito nacional se houvesse dados disponíveis.

O objetivo principal de analisar o desempenho, sob o enfoque da ecoeficiência, das operações portuárias, utilizando a Análise Relacional Grey foi atingido, permitindo uma análise de como os respectivos desempenhos poderiam ser melhorados. Como principal conclusão da aplicação realizada tem-se que o resultado em termos de ecoeficiência relacionou-se mais com alterações em impactos ambientais do que em movimentação.

A análise de sensibilidade mostra que somente alterando o grau de importância das medidas no processo de decisão, o Porto de Santos tem vantagem em relação aos demais portos, assim como os portos de Belém e Rio Grande ficaram sempre com os desempenhos mais baixos. Os desempenhos dos portos de Suape e Paranaguá/Antonina, por sua vez, parecem ter maior sensibilidade à alteração de pesos das medidas.

Como limitação relacionada a este estudo cita-se a quantidade insuficiente de dados relacionados ao meio ambiente para avaliar todos os portos. Mesmo para os portos avaliados, deveria haver indicadores mais abrangentes relacionados aos impactos ambientais, conforme mostrado na Tabela 1. Talvez, considerando outros indicadores, o ranking de desempenho fosse alterado. Outra limitação está relacionada ao fato de que os pesos e medidas foram alterados nos cenários sem o compromisso do quanto da realidade que essas mudanças representam. O intuito dessas alterações foi de entender o quanto as mudanças nas variáveis influenciariam no desempenho dos portos.

Como recomendações para outros estudos indicam-se a utilização de outros indicadores e a ampliação do número de portos, realizando pesquisa de campo mais abrangente para o levantamento de dados. Também seria indicada a utilização de outra técnica multicritério que pudesse lidar com diferenças de escala de movimentação do porto de Santos em relação aos demais. Ainda como recomendação, seria interessante uma discussão aprofundada, relacionando os indicadores e o risco ambiental que cada um representa no processo de avaliação.

Seria interessante realizar, ainda, uma análise da viabilidade das ações para melhoria da ecoeficiência dos portos, tanto do ponto de vista de aumento de valor do serviço quanto de redução de influências ambientais. Além disso, 
recomenda-se avaliar os terminais portuários de forma independente, ao invés da análise do porto como um todo, o que permitiria maior enfoque nas características próprias de cada um deles.

\section{AGRADECIMENTOS}

Os autores agradecem o apoio à pesquisa pela Fundação de Amparo à Pesquisa do Estado do Rio de Janeiro - FAPERJ e pelo Conselho Nacional de Desenvolvimento Científico e Tecnológico - CNPq.

\section{REFERÊNCIAS BIBLIOGRÁFICAS}

Agência Nacional de Transportes Aquaviários - ANTAq (2011a) O porto verde: modelo ambiental portuário / Agência Nacional de Transportes Aquaviários. - Brasília, Brasil.

Agência Nacional de Transporte Aquaviário - ANTAq (2011b) Boletim informativo portuário. Disponível em: <http://www.antaq. gov.br/Portal/pdf/BoletimPortuario/BoletimPortuarioQuartoTrim estre2011.pdf $>$. (Acesso em 05/07/2012).

Bischoff, E. (2008) Estudo da Utilização de Algorítimos Genéticos para seleção de redes de acesso. Dissertação (Mestrado). Departamento de Engenharia Elétrica. Universidade de Brasília, DF.

Britsh Petroleum (BP) (2003) Relatório anual 2003. Disponível em: <www.bp.com>. (Acesso em: 01/10/2011).

Cantarino, A. A. A. (2003) Desenvolvimento de indicadores de impacto ambiental como instrumento de gestão e controle no processo de licenciamento ambiental e empreendimentos ambientais de exploração de produtos nas áreas Offshore. Tese (Doutorado). PPE/COPPE/UFRJ, Rio de Janeiro.

Cheon, S., D. E. Dowall e D. Song (2009) Evaluating impacts of institutional reforms on port efficiency changes: Ownership, corporate structure, and total factor productivity changes of world containe ports. Transportation Research Part E, v. 46, n. 4, p. 546-561. DOI: $10.1016 /$ j.tre.2009.04.001.

Companhia Docas do Pará - CPD (2011) Estatísticas do ano de 2011. Disponível em: <http://www.cdp.com.br/estatisticas detalhes.php?nEstatisticaAno=2011>. (Acesso em: 05/07/2012).

Deng, J. (1989) Introduction to grey theory. In: Journal of Grey Systems, v.1, n. 1989, p. 1-24.

Garcia, P. A. de A., J. C. Silva Neves, C. M. C. Jacinto e E. A. L. Droguett (2005) Utilização de análise relacional grey no gerenciamento da cadeia logística. XXVII Simpósio Brasileiro de Pesquisa Operacional. Gramado, RS.

Gomes, L. F. A. M. (2004) Tomada de decisões em cenários complexos: introdução aos métodos discretos do apoio multicritério à decisão - São Paulo: Pioneira Thomson Learning.

Global Reporting Initiative (GRI) (2000) Sustainable Reporting Guidelines on Economic, Environmental and Social Performance. Global Reporting Initiative, p. 16-18, Boston, USA.

Hendriks, C. e D. Jager (2001) Economic Evaluation of Sectoral Emission Reduction Objectives for Climate Change: Economic Evaluation of Methane Emission Reduction in the Extraction, Transport and Distribution of Fossil Fuels in the EU. European Commission Studies, Holanda, 2001. Disponível em: <http://ec.europa. eu/environment/enveco/climate_change/pdf/fossilfuel.pdf $>$. (Acesso em: 01/10/ 2011).

Hsu, Y., W. Li e K. Chen (2009) Structuring critical success factors of airline safety management system using a hybrid model. Transportation Research Part E. Article in press. DOI: 10.1016/ j.tre.2009.08.005.

JOC (2012) The JOC top 50 world conteiner ports. Journal Of Comerce, v. 13, n. 29, p.24-30. Disponível em: <http://jocdigital.com/ 08202012/08202012\#\&pageSet=15>. (Acesso em: 01/05/2013).

Leal Jr, I. C. (2010) Método de Escolha Modal para transporte de produtos perigosos com base em medidas de ecoeficiência. Tese (Doutorado). PET/COPPE/UFRJ, Rio de Janeiro.

Leal Jr, I. C. e P. A. A. Garcia (2008) Utilização de análise relacional grey para seleção dos modos de transporte baseando-se na ecoeficiência. XI Encontro de Modelagem Computacional, Volta Redonda.

Leal Jr, I. C. e M. de A. D'Agosto (2009) Escolha modal para transporte de produtos perigosos com base em aspectos ambientais: caso dos modos terrestres para o etanol no Brasil. VII Rio de Transportes, Rio de Janeiro.
Leal Jr, I. C. e M. de A. D'Agosto (2011) Modal choice for transportation of hazardous materials: the case of land modes of transport of bio-ethanol in Brazil. Journal of Cleaner Production, 19 (2011) 229-240. DOI: 10.1016/j.jclepro.2010.02.006.

Leyen, B. C. (2008) Eco-eficiência na exploração e produção de petróleo e gás em regiões de florestas tropicais úmidas: o caso da Petrobrás na Amazônia. Dissertação (Mestrado). COPPE/UFRJ, Rio de Janeiro.

Lo, S., Y. Chao e G. Lee (2009) Grey Relational Evaluation on Road Project Delivery Models. AIP Conf. Proc, v. 1148, p. 480-484. DOI: 10.1063/1.3225353.

Lu, M. e K. Wevers (2007) Application of grey relational analysis for evaluating road traffic safety measures: advanced driver assistance systems against infrastructure redesign. IET Intell. Transp. Syst., v. 1, n. 1. DOI: 10.1049/iet-its:20060022.

Malczewiski, J. (1999). GIS and multicriteria decision analisys. John \& Wiley Sons. New York

Martins, A. R. S., C. Alvear e E. M. Santos (1999) Análise dos Principais Programas de Conservação de Energia no Brasil e no Exterior. Eficiência Energética - Integrando Usos e Reduzindo Desperdícios.

Michelini, A. N. C., R. F. Jappur, P. M. Selig e A. A. Leripio (2004) Uma proposta de incorporação de indicadores de ecoeficiência ao setor termoelétrico brasileiro a carvão mineral. XXIV Encontro Nac. de Engenharia de Produção, Florianópolis, SC, Brasil. Disponível em: <http://www.abepro.org.br/biblioteca/enegep2004_enegep 1003_1413.pdf $>$. (Acesso em: 01/12/2011).

Notteboom, T. E. (2004) Container Shipping and ports: an overview. Review of Network Economics. Anwerp, v. 3, n. 2, p. 86-106, junho de 2004. DOI: 10.2202/1446-9022.1045.

Pai, T., K. Hanaki, H. Ho e C. Hsieh (2007) Using grey system theory to evaluate transportation effects on air quality trends in Japan. Transportation Research Part D, v. 12, n. 3, 158-166. DOI: 10.1016/j.trd.2007.01.007.

PETROBRAS (2003) Relatório anual, análise financeira e demonstrações contábeis 2003.

Qu, L., Y. Chen e M. Yang (2007) A Dynamic Combination Forecast Model for Analysis Transport Volume Time Series. ICNC, v. 1, p.705-709, Third International Conference on Natural Computation (ICNC 2007). DOI: 10.1109/ICNC.2007.19.

Pinho, H. M., M. N. Catanzano e P. J. C. Candeira (2011) Ecoeficiência: um estudo de caso em uma indústria química. 3rd. International Workshop Advances in Cleaner Production. São Paulo, Brasil.

Santos, M. K. (2001) Eco-eficiência e avaliação dos sistemas integrados de gestão. Dissertação (mestrado). Universidade Federal do Rio Grande do Sul, Porto Alegre/RS.

Taha, H. A. (2008) Pesquisa operacional: uma visão geral. Prentice Hall, $8^{a}$ edição, São Paulo.

Valois, N. A. L. (2009) Proposição do uso de indicadores ambientais na avaliação de desempenho dos portos brasileiros. Programa de pós-graduação em engenharia mecânica. Dissertação (Mestrado). Universidade Federal de Pernambuco.

Wang, R., C. Ho, C. Feng e Y. Yang (2004) A comparative analysis of the operational performance of Taiwan's major airports. Journal of Air Transport Management, v. 10, n. 5, p. 353-360. DOI: 10.1016/j.jairtraman.2004.05.005.

Wen, K. (2004) Grey Systems: Modeling and Prediction. Printed in USA by Yang's Scientific Press.

World Business Council For Sustainable Development (WBCSD) (2000) Measuring Eco-Efficiency: A Guide to Reporting Company Performance. Geneva, Suíça.

World Business Council For Sustainable Development (WBCSD). (2006) Eco-efficiency learning module. Alemanha. Disponível em: $<$ http://www.wbcsd.org/pages/EDocument/EDocumentDetails.as px?ID=13593\&NoSearchContextKey=true $>$. (Acesso em: 01/04/2013).

Yang, C. e B. Chen (2005) Supplier selection using combined analytical hierarchy process and grey relational analysis. Journal of Manufacturing Technology Management v. 17 n. 7, p. 926-941. DOI: $\underline{10.1108 / 17410380610688241}$. 